\title{
Terapia nutricional para pacientes com Covid-19 em cuidados intensivos: Uma abordagem acerca de estudos retrospectivos
}

\author{
Nutritional therapy for patients with Covid-19 in intensive care: An approach to retrospective \\ studies \\ Terapia nutricional para pacientes con Covid-19 en cuidados intensivos: Un acercamiento a los \\ estudios retrospectivos
}

Jardel Alves da Costa

ORCID: https://orcid.org/0000-0002-9844-0770 Universidade Federal do Piauí, Brasil

E-mail: jardelalves@ufpi.edu.br

Diêgo de Oliveira Lima

ORCID: https://orcid.org/0000-0001-8211-9416 Universidade Federal do Piauí, Brasil E-mail: diego.oliveira@ufpi.edu.br

Iara Pereira Melo Moreira

ORCID: https://orcid.org/0000-0003-3077-7577

Centro Universitário Santo Agostinho, Brasil E-mail: iaramelo10@hotmail.com

Beatriz Souza Santos

ORCID: https://orcid.org/0000-0002-2464-4674 Universidade Federal do Piauí, Brasil E-mail: santosbsnutri@gmail.com

Fatima Rosane Barros

ORCID: https://orcid.org/0000-0003-1383-076X Universidade Federal do Piauí, Brasil E-mail: rosa_barros18@ufpi.edu.br

Tamiris Ramos Silva

ORCID: https://orcid.org/0000-0003-3433-4052 Universidade Federal do Piauí, Brasil

E-mail: tamirisramos2016@ hotmail.com

Gabrielly Martins de Barros

ORCID: https://orcid.org/0000-0001-9696-424X Faculdade Uninassau, Brasil E-mail: gabyrhcp00@hotmail.com

Edna Lara Vasconcelos Da Silva Gomes ORCID: https://orcid.org/0000-0003-0234-4862 Centro Universitário Santo Agostinho, Brasil E-mail: ednalara_vas@hotmail.com Amanda Raquel Silva Sousa ORCID: https://orcid.org/0000-0002-1578-4624 Faculdade de Floriano, Brasil E-mail: amandasousa1r@gmail.com

Renata Rodrigues Costa Fontinele ORCID: https://orcid.org/0000-0002-6121-0322 Universidade Federal do Piauí, Brasil

E-mail: renatafontinele25@hotmail.com

Fernanda Kassiely de Sousa Veloso ORCID: https://orcid.org/0000-0002-5289-2624 Universidade Federal do Piauí, Brasil E-mail: f.kassiely@gmail.com

Rita de Cássia Moura da Cruz ORCID: https://orcid.org/0000-0002-9721-0535 Universidade Federal do Piauí, Brasil E-mail:ritamoura.96@gmail.com

Ariane de Oliveira Lima Sousa ORCID: https://orcid.org/0000-0002-4313-4346 Universidade Federal do Piauí, Brasil E-mail: arianelima.nutri@outlook.com 
Dayane Dayse de Melo Costa

ORCID: https://orcid.org/0000-0001-5635-5183 Universidade Federal do Piaú, Brasil E-mail: dayane785@hotmail.com

Mikaella Isis de Macedo

ORCID: https://orcid.org/0000-0002-1799-2064 Universidade Federal do Piaú, Brasil

E-mail: mikaellaisisdemacedo@ hotmail.com

Lucineide de Brito Rocha

ORCID: https://orcid.org/0000-0003-0832-5507 Universidade Federal do Piaú, Brasil E-mail: lucineidedebrito@gmail.com

Elissandra de Carvalho Ramos

ORCID: https://orcid.org/0000-0001-7740-0182 Centro Universitário Santo Agostinho, Brasil E-mail: elissandraramos123@gmail.com

Clécia Maria da Silva

ORCID: https://orcid.org/0000-0002-0830-7432 Universidade Federal do Piauí, Brasil E-mail: Cleciasilva123silva@gmail.com

Lívia Carvalho Taveira

ORCID: https://orcid.org/0000-0001-6650-3047 Universidade Federal do Piauí, Brasil E-mail: livia-taveira@hotmail.com

Nara Vanessa Barros dos Anjos

ORCID: https://orcid.org/0000-0003-2044-7064 Universidade Federal do Piauí, Brasil E-mail: nara.vanessa@hotmail.com

\title{
Resumo
}

Esta pesquisa objetivou revisar a literatura acerca de estudos retrospectivos realizados no âmbito da pandemia da Covid-19, que relataram sobre a terapia nutricional utilizada para pacientes portadores da doença internados em Unidades de Terapia Intensiva (UTI). A revisão integrativa foi realizada a partir de buscas nos bancos de dados eletrônicos acerca de publicações de artigos originais escritos em inglês e português, entre os anos de 2019 a 2021. As buscas foram realizadas nas bases de dados: Science Direct (Biblioteca virtual da Elsevier), Scielo (Scientific Electronic Library Online), Pubmed/Medline (Medical Literature Analysis and Retrievel System Online) e BVS (Biblioteca Virtual em Saúde), utilizando como descritores as palavras SARS-CoV-2, Nutrição Enteral, Nutrição Parenteral e Unidade de Terapia Intensiva, nos idiomas inglês e português. Após o processo de busca e seleção utilizando os critérios citados na metodologia, foram selecionados 3 artigos. A terapia nutricional é parte fundamental do processo de recuperação dos pacientes críticos com Covid-19, já que no âmbito do período de internação o processo de desnutrição é acelerado, devido a característica clínica hipercatabólica da doença relacionada a distúrbios metabólicos. Algumas diretrizes acerca da terapia nutricional para pacientes com Covid-19 já foram criadas a partir de declarações de profissionais vinculados as sociedades de nutrição clínica, e as recomendações tem sido bastante utilizadas como princípios terapêuticos nos centros médicos de cuidado aos pacientes neste estado patológico.

Palavras-chave: SARS-CoV-2; Nutrição enteral; Nutrição parenteral; Unidade de terapia intensiva.

\begin{abstract}
This research aimed to review the literature about retrospective studies carried out within the scope of the Covid-19 pandemic, which reported on the nutritional therapy used for patients with the disease admitted to Intensive Care Units. The integrative review was carried out from searches in electronic databases about publications of original articles written in English and Portuguese, between the years 2019 to 2021. The searches were carried out in the databases: Science Direct (Elsevier virtual library), Scielo (Scientific Electronic Library Online), Pubmed / Medline (Medical Literature Analysis and Retrievel System Online) and VHL (Virtual Health Library), using the words SARS-CoV-2, Enteral Nutrition, Parenteral Nutrition and Health Unit as descriptors. Intensive Care, in English and Portuguese. After the search and selection process using the criteria cited in the methodology, 3 articles were selected. Nutritional therapy is a fundamental part of the recovery process for critical patients with Covid-19, since within the hospitalization period the malnutrition process is accelerated, due to the hypercatabolic clinical characteristic of the disease related to metabolic disorders. Some guidelines about nutritional therapy for patients with Covid-19 have already been created from statements by professionals linked to clinical nutrition societies, and the recommendations have been widely used as therapeutic principles in medical care centers for patients in this pathological state.
\end{abstract}

Keywords: SARS-CoV-2; Enteral nutrition; Parenteral nutrition; Intensive care unit.

\section{Resumen}

Esta investigación tuvo como objetivo revisar la literatura sobre estudios retrospectivos realizados en el ámbito de la pandemia Covid-19, que informaron sobre la terapia nutricional utilizada en pacientes con la enfermedad ingresados 
en Unidades de Cuidados Intensivos. La revisión integradora se realizó a partir de búsquedas en bases de datos electrónicas sobre publicaciones de artículos originales escritos en inglés y portugués, entre los años 2019 a 2021 . Las búsquedas se realizaron en las bases de datos: Science Direct (biblioteca virtual de Elsevier), Scielo ( Scientific Electronic Library Online), Pubmed / Medline (Medical Literature Analysis and Retrievel System Online) y VHL (Virtual Health Library), utilizando como descriptores las palabras SARS-CoV-2, Enteral Nutrition, Parenteral Nutrition and Health Unit. Intensive Care, en Inglés y portugués. Tras el proceso de búsqueda y selección utilizando los criterios citados en la metodología, se seleccionaron 3 artículos. La terapia nutricional es parte fundamental del proceso de recuperación de los pacientes críticos con Covid-19, ya que dentro del período de hospitalización se acelera el proceso de desnutrición, debido a la característica clínica hipercatabólica de la enfermedad relacionada con los trastornos metabólicos. Algunas pautas sobre terapia nutricional para pacientes con Covid-19 ya han sido elaboradas a partir de declaraciones de profesionales vinculados a sociedades de nutrición clínica, y las recomendaciones han sido ampliamente utilizadas como principios terapéuticos en los centros de atención médica para pacientes en este estado patológico.

Palabras clave: SARS-CoV-2; Nutrición enteral; Nutrición parenteral; Unidad de terapia intensiva.

\section{Introduçãa}

A nova Síndrome Respiratória Aguda Grave Coronavírus 2 (SARS-CoV-2), responsável pela doença coronavírus 2019 (COVID-19) foi identificada pela primeira vez em humanos no final de 2019 em Wuhan, China. Desde então, gradualmente se espalhou pelo mundo, a tal ponto que, em março de 2020, a Organização Mundial da Saúde (OMS) declarou a COVID-19 uma pandemia global (Lai, et al., 2020; Abd El-Aziz \& Stockand, 2020).

Os pacientes críticos internados em Unidade de Terapia Intensiva (UTI) têm maior risco de desnutrição com redução da massa muscular e funcionalidade do músculo esquelético, causando prejuízo na qualidade de vida dos pacientes, levando a incapacidade e impactando em maior morbidade. O impacto desses aspectos é proporcional ao tempo de internação e se estende mesmo após a alta hospitalar (McClave et al., 2016; Van Zanten, De Waele \& Wischmeyer, 2019; Singer et al., 2019).

Pacientes afetados pela Covid-19 podem estar em risco de desnutrição devido a ingestão reduzida de alimentos, catabolismo acelerado relacionado à inflamação, mobilidade reduzida devido à internação prolongada, bem como idade avançada e comorbidades. Além disso, na presença de infecção por SARS-CoV-2, o desenvolvimento de inflamação e sepse podem contribuir para exacerbar essas alterações (Barazzoni et al., 2020; Singer et al., 2019; Gomes et al., 2018; Volkert et al., 2019).

O tratamento de pacientes críticos com Covid-19 é um processo longo e o suporte nutricional é parte importante desse processo. O papel da nutrição é ainda mais realçado em pacientes internados em UTI, devido à gravidade da insuficiência respiratória (Bharadwaj et al., 2016; Wu et al., 2020; Maggini et al., 2007; Barazzoni et al., 2020).

Dessa maneira, o cuidado nutricional ideal associado à terapia de suporte de vida em UTI tem potencial para otimizar o prognóstico de pacientes afetados pela Covid-19 (Zhu et al., 2020). A intervenção precoce com terapia nutricional adequada ao estado clínico, considerando os aspectos multifatoriais da desnutrição e que atenda às necessidades nutricionais do paciente pode minimizar os efeitos metabólicos provocado pelo SARS-CoV-2, reduzir o dano oxidativo celular, regular a resposta imune, além de minimizar o catabolismo e degradação muscular (Li et al., 2020; Santos et al., 2021).

Diante do contexto relatado, esta pesquisa objetivou revisar a literatura acerca de estudos retrospectivos realizados no âmbito da pandemia da Covid-19, que relataram sobre a terapia nutricional utilizada para pacientes portadores da doença internados em Unidades de Terapia Intensiva.

\section{Metodologia}

A revisão integrativa foi realizada a partir de buscas nos bancos de dados eletrônicos acerca de publicações de artigos originais escritos em inglês e português, entre os anos de 2019 a 2021. As buscas foram realizadas nas bases de dados: Science Direct (Biblioteca virtual da Elsevier), Scielo (Scientific Electronic Library Online), Pubmed/Medline (Medical Literature 
Analysis and Retrievel System Online) e BVS (Biblioteca Virtual em Saúde), utilizando como descritores as palavras SARSCoV-2, Nutrição Enteral, Nutrição parenteral, Unidade de Terapia Intensiva, nos idiomas inglês e português, utilizando o termo "and" como operador boleano.

Além da utilização dos descritores, a seleção dos estudos ocorreu de acordo com os critérios de inclusão que foram: artigos originais completos que realizaram observações retrospectivas da terapia nutricional utilizada para pacientes com Covid-19 em cuidados intensivos. Os trabalhos devem ter sido publicados entre os anos de 2019 a 2021 nos idiomas inglês e português, com a presença dos referidos descritores. Foram excluídos todos os artigos que não corroboravam com o objetivo da pesquisa após leitura dos resumos de cada estudo, e aqueles que não se enquadraram nos critérios de inclusão. O processo de busca e seleção sistemática dos estudos está representado no Quadro 1.

Quadro 1. Busca e seleção dos estudos.

\begin{tabular}{|c|c|c|c|}
\hline \multicolumn{4}{|c|}{ Busca utilizando descritores } \\
\hline Base & Idioma & Descritores & Resultados \\
\hline \multirow[t]{2}{*}{ Pubmed } & Português & \multirow{8}{*}{$\begin{array}{l}\text { Covid 19, Terapia } \\
\text { nutricional, } \\
\text { Unidade de terapia } \\
\text { intensiva, estudo } \\
\text { retrospectivo. }\end{array}$} & 0 \\
\hline & Inglês & & 184 \\
\hline \multirow[t]{2}{*}{ Scielo } & Português & & 0 \\
\hline & Inglês & & 0 \\
\hline \multirow[t]{2}{*}{ Science direct } & Português & & 0 \\
\hline & Inglês & & 197 \\
\hline \multirow[t]{2}{*}{ BVS } & Português & & 0 \\
\hline & Inglês & & 1 \\
\hline \multicolumn{4}{|c|}{ Aplicação dos critérios de inclusão e exclusão } \\
\hline Base & Idioma & Resultado & TOTAL \\
\hline \multirow[t]{2}{*}{ Pubmed } & Português & 0 & \multirow[t]{2}{*}{1} \\
\hline & Inglês & 1 & \\
\hline \multirow[t]{2}{*}{ Scielo } & Português & 0 & \multirow[t]{2}{*}{0} \\
\hline & Inglês & 0 & \\
\hline \multirow[t]{2}{*}{ Science direct } & Português & 0 & \multirow[t]{2}{*}{1} \\
\hline & Inglês & 1 & \\
\hline \multirow[t]{2}{*}{ BVS } & Português & 0 & \multirow[t]{2}{*}{1} \\
\hline & Inglês & 1 & \\
\hline
\end{tabular}

Fonte: Autores.

\section{Resultados e Discussão}

Após o processo de busca e seleção utilizando os critérios citados na metodologia, foram selecionados 3 artigos. Na Tabela 1 é possível visualizar os autores, metodologia e principais resultados relatados por cada estudo. 
Tabe1a 1. Descrição dos estudos selecionados.

\begin{abstract}
Estudo 1: Formisano et al. 2021
Descrição metodológica: Foram incluídos no estudo 49 pacientes com covid-19 internados na UTI. Os 49 receberam terapia nutricional enteral (NE) com suporte nasogástrico e nutrição parenteral (NP) suplementar em via periférica. As necessidades de energia foram estimadas usando a equação de Harris-Benedict multiplicada por um fator de correção de 1,2 ou 1,3 para a presença da doença aguda. O manejo dos pacientes críticos foi realizado considerando o dispositivo específico utilizado para o suporte respiratório, onde pacientes em sub-UTI com a "Submask" (uma máscara de mergulho especialmente modificada conectada a um ventilador), com um nível de saturação de oxigênio que pode permitir o descolamento da "Submask" e o posicionamento temporário da cânula nasal com alta fluxo de oxigênio, por 10 a 15 minutos, nos quais os pacientes recebiam NE com fórmula básica (1477 kcal - porcentagem de proteínas: 19\% da kcal total; porcentagem de lipídios: $45 \%$ da kcal total; porcentagem de carboidratos: $36 \%$ da kcal total) e NP periférica suplementar. Os pacientes tratados com ventilação não invasiva (VNI) através do capacete elmo, receberam dois suplementos nutricionais orais líquidos de alto teor calórico e alto teor de proteínas insípidos $(200 \mathrm{~mL}$; $320 \mathrm{kcal} ; 20 \mathrm{~g}$ de proteínas) por dia em bolus (grande quantidade) com uma seringa de $500 \mathrm{~mL}$ e NP periférica suplementar, se tolerada. As fórmulas de NE eram pobres em carboidratos (composição de $29 \%$ a $39 \%$ de carboidratos, de $18 \%$ a $37 \%$ de proteínas, de 500 a $750 \mathrm{kcal}$, em $500 \mathrm{~mL}$ ).

Principais resultados relatados: $O$ tratamento nutricional prescrito foi bem tolerado, no entanto, algumas complicações gastrointestinais foram observadas (saciedade precoce, diarreia, distensão abdominal). 46,9\% dos pacientes da UTI receberam alta hospitalar; enquanto 53,1\% dos pacientes da UTI morreram.
\end{abstract}

Estudo 2: Alonso, Hernández \& Crespo, 2020

Descrição metodológica: Foram incluídos no estudo 20 pacientes com covid-19 internados na UTI. 14 pacientes receberam NE com suporte orogástrico e NP complementar com suporte via central. E os 6 restantes receberam NE exclusiva. Todos os pacientes foram intubados durante a admissão na UTI, para início da ventilação mecânica. 70\% dos pacientes necessitaram de NE + NP e 30\% exclusivamente NE. A prescrição de calorias e proteínas foram feitas de acordo com as recomendações das diretrizes clínicas da Sociedade Europeia de Nutrição Clínica e Metabolismo (ESPEN) e Sociedade Americana de Nutrição Parenteral e Enteral (ASPEN), onde as necessidades de energia calculadas para pacientes com menos de 60 anos de idade foram $1.800 \mathrm{kcal} /$ dia e $1.535 \mathrm{kcal} /$ dia para maiores de 60 anos. A composição das fórmulas utilizadas foram: em 5\% de normocalórica-hiperproteica, em 5\% de normocalórica-normoproteica, em $15 \%$ de imunonutrição hipercalórica (ácidos graxos $\omega$ - 3) e 75\% hipercalórico e hiperproteica; sendo $89 \%$ dessas fórmulas com fibras. Todos os pacientes que receberam NP tiveram a seguinte composição da fórmula dietética: hiperproteica-hipercalórica com as seguintes quantidades médias de macronutrientes / dia: 11,8 g de nitrogênio (73,8 g de proteínas), $135 \mathrm{~g}$ de glicose e 45,5 g de lipídeos.

Principais resultados relatados: Apenas $30 \%$ dos indivíduos tiveram tolerância total à NE, desta maneira a NP foi iniciada de forma complementar em $70 \%$ dos indivíduos. O estudo não relatou complicações gastrointestinais específicas. 50\% dos pacientes da UTI receberam alta hospitalar, enquanto $50 \%$ dos pacientes da UTI morreram.

Estudo 3: Osuna-Padilla et al. 2021

Descrição metodológica: Foram incluídos no estudo 52 pacientes com covid-19 internados na UTI. Todos receberam terapia nutricional enteral com suporte orogástrico. Os indivíduos analisados também estavam sendo ventilados mecanicamente, porém o suporte respiratório específico não foi distinguido. A colocação de sonda orogástrica foi utilizada em relação a outros acessos de curto prazo como prática institucional comum para evitar epistaxe e infecção sinusal. A prescrição de calorias e proteínas foram feitas de acordo com as recomendações da Sociedade Americana de Nutrição Parenteral e Enteral (ASPEN) e da Sociedade Europeia de Nutrição Clínica e Metabolismo (ESPEN), com uma meta geral de $25 \mathrm{kcal} / \mathrm{kg} / \mathrm{dia}$ e $1,3 \mathrm{~g} / \mathrm{kg} / \mathrm{dia} \mathrm{de}$ proteína, respectivamente. A composição da fórmula utilizada não foi detalhada como nos estudos anteriores.

Principais resultados relatados: $O$ tratamento nutricional prescrito foi bem tolerado, no entanto, algumas complicações gastrointestinais foram observadas em 35\% dos indivíduos (diarreia, vómitos e gastroparesia). A incidência de constipação foi encontrada em $87 \%$ dos pacientes. $43 \%$ dos pacientes da UTI receberam alta hospitalar; enquanto $57 \%$ dos pacientes da UTI morreram. 
De acordo com Patel, Martindale e McClave (2020), as recomendações para terapia nutricional no indivíduo com COVID-19 seguem os mesmos princípios para a nutrição de cuidados intensivos em geral para qualquer paciente de UTI, como observado nos estudos elegidos para compor a revisão. A NE é preferível a NP, desde que o paciente esteja apto a receber nutrientes pelo trato gastrointestinal. A NE intragástrica deve ser iniciada entre 24 a 48 horas, após a admissão na UTI, em pacientes hemodinamicamente compensados, especialmente se o paciente precisar de intubação e ventilação mecânica.

Shang et al. (2020) corroboram com as informações anteriores, afirmando que a NE é a via preferencial de alimentação para pacientes gravemente enfermos que requerem terapia de suporte nutricional e não podem utilizar a via oral para alimentação, e que a NE deve ser iniciada precocemente, nas 24 horas após a admissão na UTI.

A Associação Médica da Sociedade de Nutrição Parenteral e Enteral da China recomendou o método de cinco etapas para implementar a terapia nutricional para pacientes com COVID-19, estas 5 etapas consistem em: dieta elementar, educação nutricional, suplemento nutricional oral, alimentação por sonda para NE e NP suplementar, e nutrição enteral total (NET) ou nutrição parenteral total (NPT) a depender do estado clínico do paciente (Elke et al., 2019).

A Sociedade Europeia de Nutrição Clínica e Metabolismo (ESPEN) e Sociedade Americana de Nutrição Parenteral e Enteral (ASPEN) desenvolveram diretrizes de manejo nutricional em pacientes com COVID-19. Os princípios consistem nas seguintes recomendações que corroboram com os estudos supracitados e além da indicação do suporte nutricional, também traz as recomendações de calorias e nutrientes (Barazzoni et al., 2020; Martindale, 2020).

As duas sociedades (ESPEN e ASPEN) preconizam que se deve iniciar a NE nas primeiras 24-48 horas após a admissão na UTI. A NP é uma alternativa quando o paciente não tolera NE ou é insuficiente para atender às necessidades calóricas específicas de cada paciente. A primeira opção para administração de NE é por sonda nasogástrica. Em caso de intolerância, será utilizada a via pós-pilórica. A administração por infusão contínua é preferível à administração em bolus. Iniciar NE hipercalórica-hiperproteica com fibras em baixas doses e progredir até atingir doses completas na primeira semana (meta mínima de $20 \mathrm{kcal} / \mathrm{kg} / \mathrm{dia}$ ). Em pacientes com intolerância gastrointestinal, a NE deve ser descontinuada e o uso de NP deve ser considerado, após a ineficácia dos fármacos procinéticos e desde que o paciente esteja estável hemodinamicamente (Barazzoni et al., 2020; Martindale, 2020).

Os pacientes devem então ser monitorados quanto à tolerância como feito por Osuna-Padila et al. (2021). Uma vez que a NE é iniciada com doses tróficas, ela avança lentamente durante a primeira semana para atingir a meta de fornecimento de energia e proteínas.

De acordo com Barazzoni et al. (2020), os sintomas gastrointestinais devem ser observados como indicadores de intolerância ao suporte nutricional, causados por fatores metabólicos ou mecânicos, pois estes podem prejudicar a ingestão ou absorção de alimentos, por exemplo, disfagia, náusea, vômito, diarreia, prisão de ventre ou dor abdominal. A assimilação reduzida de alimentos / nutrientes está associada a distúrbios de má absorção, como síndrome do intestino curto, insuficiência pancreática e após cirurgia bariátrica. Também está associada a distúrbios como estenoses esofágicas, gastroparesia, íleo paralítico, pseudo-obstrução intestinal, além do uso de drogas vasoativas.

A evidência de intolerância pode limitar ou até mesmo contraindicar o uso do trato gastrointestinal abrigando um maior perigo de complicações, como intestino isquêmico e a necessidade de colocação de tubo pós-pilórico. Dessa forma, fazem-se necessárias vias alternativas de nutrição, uma vez tomada a decisão da administração de NP para o paciente com COVID - 19, devido intolerância a NE, e deve seguir os princípios usuais para nutrição em cuidados intensivos (Patel, Martindale \& McClave, 2020). 


\section{Considerações Finais}

A terapia nutricional é parte fundamental do processo de recuperação dos pacientes críticos com Covid-19, já que no âmbito do período de internação o processo de desnutrição é acelerado, devido a característica clínica hipercatabólica da doença relacionada a distúrbios metabólicos, levando a piora do prognóstico desses indivíduos. Desta maneira, a terapia nutricional destinada a estes indivíduos deve ser analisada de forma eficaz, com intuito de promover o mais adequado suporte de acordo com o estado clínico do paciente.

Algumas diretrizes acerca da terapia nutricional para pacientes com covid-19 já foram criadas a partir de declarações de profissionais vinculados as sociedades de nutrição clínica, e as recomendações tem sido bastante utilizadas como princípios terapêuticos nos centros médicos de cuidado aos pacientes neste estado patológico. Dessa forma, mais estudos devem ser realizados, com finalidade de fortalecer as evidências disponíveis, assim como promover a construção de novas alternativas para um tratamento nutricional cada vez mais eficiente para os indivíduos em cuidados intensivos devido a Covid-19.

\section{Referências}

El-Aziz, T. M., \& Stockand, J. D. (2020). Recent progress and challenges in drug development against COVID-19 coronavirus (SARS-CoV-2)-an update on the status. Infection, Genetics and Evolution, 83(1), 104327.

Alonso, R., Hernández, M., JC, P. P., \& Crespo, B. (2020). Abordaje nutricional en pacientes críticos diagnosticados de neumonía por COVID-19. Ofil-Ilaphar, 1(1), 1-4.

Barazzoni, R., Bischoff, S. C., Breda, J., Wickramasinghe, K., Krznaric, Z., Nitzan, D., \& Singer, P. (2020). ESPEN expert statements and practical guidance for nutritional management of individuals with SARS-CoV-2 infection. Espen, 39(6), 1631-1638.

Bharadwaj, S., Ginoya, S., Tandon, P., Gohel, T. D., Guirguis, J., Vallabh, H., \& Hanouneh, I. (2016). Malnutrition: laboratory markers vs nutritional assessment. Gastroenterology report, 4(4), 272-280.

Elke, G., Hartl, W. H., Kreymann, K. G., Adolph, M., Felbinger, T. W., Graf, T., \& Bischoff, S. C. (2019). Clinical nutrition in critical care medicineGuideline of the German Society for Nutritional Medicine (DGEM). Clinical nutrition ESPEN, 33, 220-275.

Formisano, E., Di Maio, P., Ivaldi, C., Sferrazzo, E., Arieta, L., Bongiovanni, S., \& Demontis, S. (2021). Nutritional therapy for patients with coronavirus disease 2019 (COVID-19): Practical protocol from a single center highly affected by an outbreak of the novel severe acute respiratory syndrome coronavirus 2 (SARS-CoV-2) infection. Nutrition (Burbank, Los Angeles County, Calif.), 82, 111048.

Gomes, F., Schuetz, P., Bounoure, L., Austin, P., Ballesteros-Pomar, M., Cederholm, T., \& Bischoff, S. C. (2018). ESPEN guidelines on nutritional support for polymorbid internal medicine patients. Clinical nutrition, 37(1), 336-353.

Lai, C. C., Shih, T. P., Ko, W. C., Tang, H. J., \& Hsueh, P. R. (2020). Severe acute respiratory syndrome coronavirus 2 (SARS-CoV-2) and coronavirus disease-2019 (COVID-19): The epidemic and the challenges. International journal of antimicrobial agents, $55(3), 105924$.

Maggini, S., Wintergerst, E. S., Beveridge, S., \& Hornig, D. H. (2007). Selected vitamins and trace elements support immune function by strengthening epithelial barriers and cellular and humoral immune responses. British Journal of Nutrition, 98(S1), S29-S35.

Martindale, R. Mcclave Md. (2020). Nutrition Therapy in the Patient with COVID-19 Disease Requiring ICU Care. Journal of parenteral and enteral nutrition, 44(1), 1174-1184.

McClave, S. A., Taylor, B. E., Martindale, R. G., Warren, M. M., Johnson, D. R., Braunschweig, C., \& Compher, C. (2016). Guidelines for the provision and assessment of nutrition support therapy in the adult critically ill patient: Society of Critical Care Medicine (SCCM) and American Society for Parenteral and Enteral Nutrition (ASPEN). JPEN. Journal of parenteral and enteral nutrition, 40(2), 159-211.

Osuna-Padilla, I., Rodríguez-Moguel, N. C., Aguilar-Vargas, A., \& Rodríguez-Llamazares, S. (2021). Safety and tolerance of enteral nutrition in COVID-19 critically ill patients, a retrospective study. Clinical Nutrition ESPEN. 1 (1), 1-6.

Patel, J. J., Martindale, R. G., \& McClave, S. A. (2020). Relevant Nutrition Therapy in COVID-19 and the Constraints On Its Delivery by a Unique Disease Process. Nutrition in Clinical Practice, 35(5), 792-799.

Santos, B.S., Marreiros, C.S., Veloso, F.K. de S., Costa, J.A. da.; Ferreira, Kr.; Carvalho, L.de.; Fontinele, R. R. C.; Silva, S. D. da., Rodrigues, G. P. (2021). Terapia nutricional em pacientes com COVID-19: algumas considerações e evidências científicas. Reaseach, Society and Devolupment, 10 (3), e41210313400.

Shang, Y., Pan, C., Yang, X., Zhong, M., Shang, X., Wu, Z., \& Chen, D. (2020). Management of critically ill patients with COVID-19 in ICU: statement from front-line intensive care experts in Wuhan, China. Annals of intensive care, 10(1), 1-24.

Singer, P., Blaser, A. R., Berger, M. M., Alhazzani, W., Calder, P. C., Casaer, M. P., \& Bischoff, S. C. (2019). ESPEN guideline on clinical nutrition in the intensive care unit. Clinical nutrition, 38(1), 48-79. 
Research, Society and Development, v. 10, n. 5, e24810514861, 2021

(CC BY 4.0) | ISSN 2525-3409 | DOI: http://dx.doi.org/10.33448/rsd-v10i5.14861

Van Zanten, A. R. H., De Waele, E., \& Wischmeyer, P. E. (2019). Nutrition therapy and critical illness: practical guidance for the ICU, post-ICU, and longterm convalescence phases. Critical Care, 23(1), 1-10.

Volkert, D., Beck, A. M., Cederholm, T., Cruz-Jentoft, A., Goisser, S., Hooper, L., \& Bischoff, S. C. (2019). ESPEN guideline on clinical nutrition and hydration in geriatrics. Clinical nutrition, 38(1), 10-47.

Wu, C., Chen, X., Cai, Y., Zhou, X., Xu, S., Huang, H., \& Song, Y. (2020). Risk factors associated with acute respiratory distress syndrome and death in patients with coronavirus disease 2019 pneumonia in Wuhan, China. JAMA internal medicine, 180(7), 934-943.

Zhu, N., Zhang, D., Wang, W., Li, X., Yang, B., Song, J., \& Tan, W. (2020). A novel coronavirus from patients with pneumonia in China, 2019. New England journal of medicine. 20(1), 727-733. 